\title{
A minor perturbing effect of retinal locus on dot pattern recognition: Rejection of a possible artifact $* * *$
}

\author{
WILLIAM R. UTTAL \\ University of Michigan, Ann Arbor, Mich. 41804
}

An empirical test of the influence of retinal locus in a dot masking experiment shows it to be a minor perturbing effect about one-tenth of the magnitude of the organizational effects under the worst conditions.

In an earlier study of pattern recognition using a dot masking paradigm (Uttal, 1971) it was observed that the dots making up the corners of a triangle contributed less to the recognition of that geometric form than did the constituent dots of the sides. Specifically, when the dots making up the sides were omitted from the dotted triangle, the effect, as measured by the percentage of the stimuli correctly oriented, was $15 \%$ to $30 \%$ lower, depending on the masking dot density, than when the same number of dots was omitted from the corners. It was concluded that this effect was due to the differing geometrical role played by the dots of the sides and corners, respectively.

However, it may also be noted (see Fig. 1) that the average distance of the omitted dots on the sides from the center of the triangle was about $13 / 4 \mathrm{deg}$, while the average distance of the dots of the corners from the center

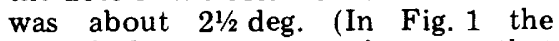
circled dots represent the ones that were omitted in the original experiment-the sides being omitted in certain trials and the corners in others.) Since it is known that, under certain conditions, retinal locus may play an important role in form recognition (Hershenson, 1969; Menzer \& Thurmond, 1970), the possibility that retinal locus or some difference in acuity as a function of locus might have substantially biased our data must be considered.

The purpose of this present paper is to consider if differing retinal acuity or any other locus effect on pattern recognition did, indeed, play any significant role in the earlier study (Uttal, 1971). The results of an experiment specifically designed to test for a retinal locus effect are

*Reprint requests should be addressed to: W. R. Uttal, 1052 MHRI, University of Michigan, Ann Arbor, Mich. 48104.

**I would like to acknowledge the support I have received for this research program in the form of a Research Scientist Award from the National Institute of Mental Health (1-K05-MH29941) and from a research grant from the National Science Foundation (GB25431). reported. The results of the experiment suggest that locus is not an important variable over the range of loci and within the context of the experimental paradigm we have been using.

Before we discuss the details of the present experiment, let us consider the possible relevance of visual acuity per se to this problem. Some readers have suggested that, since it is known that acuity varies as a function of retinal locus, perhaps difficulty in distinguishing between adajcent dots affected the pattern recognition task in our triangle experiments. Such a locus-specific acuity argument may be disposed of immediately. Acuity at 1 , 2 , and $3 \mathrm{deg}$ off the line of sight is

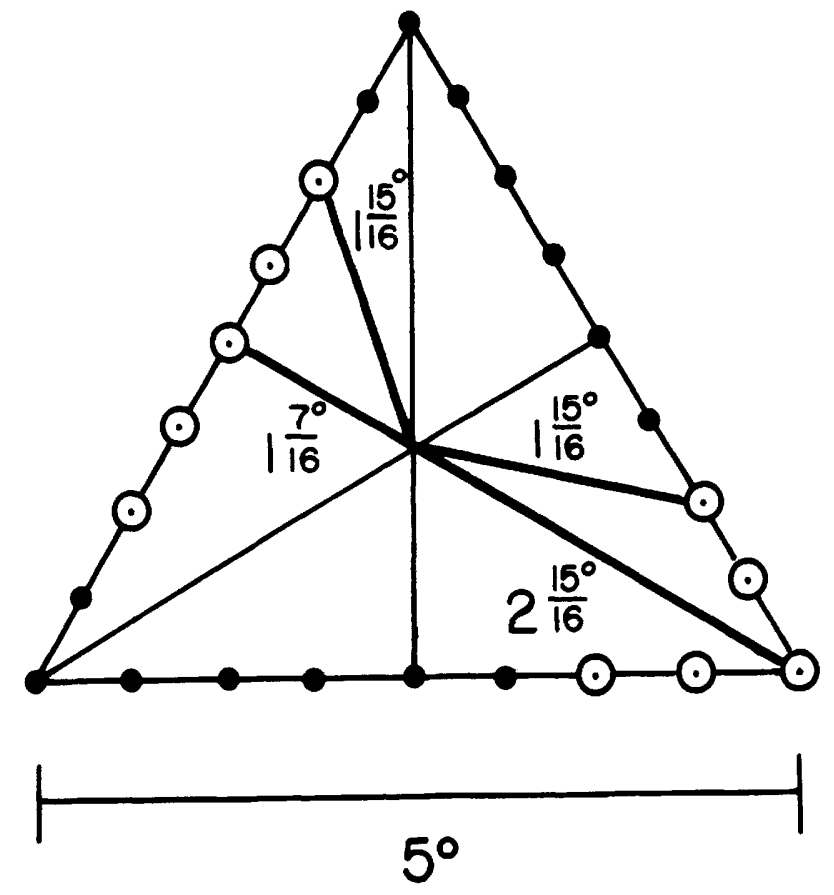

Fig. 1. A construction showing the dimensions of the two types of dotted triangles used in the earlier experiment (Uttal, 1971). Each black dot is the potential locus of one of the dots of the complete triangle. The line of five circled dots is one of three sides absent in the Type III triangle, while the corner configuration of five circled dots is one of three such corners absent in a Type II triangle. The four heavier lines represent the visual angle in degrees (indicated as an approximating fraction) of the extreme points for each type of triangle. 


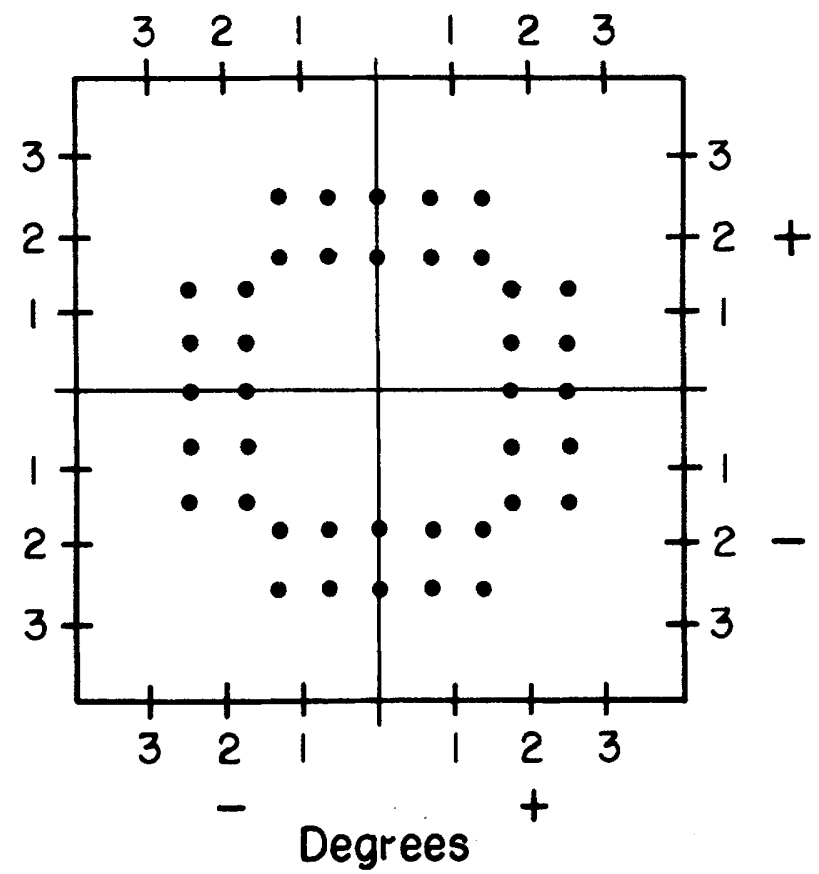

Fig. 2. A plan of the eight stimuli, each consisting of a line of five dots, used in the present experiment to show that retinal locus is only a minor perturbing factor in this particular experimental paradigm. In the actual experiment, only one of the dotted patterns and a preliminary alerting point appeared on the oscilloscope.

during the first second of the DVN. It seemed, at first, highly unlikely that the accuracy of placement of the line of fixation in this moving field would have been sufficient to allow a substantial retinal locus effect to bias our results over the $3 / 4$-deg average difference of stimulus dot locations of the corners and sides of the stimulus triangle.

But unlikely as it may have initially seemed, it is best to answer this question with an appropriate empirical test rather than to simply reassert the a priori analysis.

\section{Methods and Results}

Seven undergraduate student Ss with normal or corrected vision were run in an experiment that was exactly like the original triangle experiment, except that the stimuli were dotted lines chosen from among the set shown in Fig. 2. Each stimulus line was composed of five dots separated by $40 \mathrm{~min}$ of visual angle. Four horizontal lines were placed at $+2 \frac{1}{2}$, $+1 \frac{3 / 4}{}, \quad-13 \frac{3}{4}, \quad-2 \frac{1 / 2}{\mathrm{deg}}$ vertical displacement from the center of the masking noise, and four vertical lines were placed at equivalent displacements horizontally. These values were chosen as the closest approximations to the nominal average values of the dots of the sides and corners, respectively, in the triangle experiment. DVN of $1 \mathrm{sec}$ duration was presented both prior to and following each stimulus line. DVN levels (the time between sequential noise dots) of 4,3 , and $2 \mathrm{msec}$ were chosen so that the data could be directly compared with the previous experiments. A ready signal identical to those used in the previous experiment was used. All other

Table 1

(A) The results of the present experiment are tabulated as percent correct recognition scores for each of the two visual angles as a function of the density of the dynamic visual noise. Clearly, there is virtually no retinal locus effect at 4 or $3 \mathrm{msec}$ DVN and only a small effect at the denser 2-msec level. Chance scores in the experiment would be $50 \%$. (B) The results of the original triangle experiment (Uttal, 1971) are tabulated in a mannex corresponding to Part (A) of this table. Clearly, the effect of triangle type is large and, in the worst case (2 msec), 10 times larger than the retinal locus effect. Chance scores in this experiment would be $25 \%$.

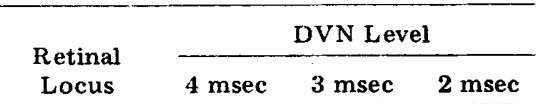

(A) Results of Current Experiment

\begin{tabular}{|c|c|}
\hline 13/4 Deg & 91.1 \\
\hline
\end{tabular}

$\begin{array}{lllll}21 / 2 & \text { Deg } & 90.5 & 81.8 & 72.3\end{array}$

\begin{tabular}{llll}
\hline & \multicolumn{3}{c}{ DVN Level } \\
\cline { 2 - 4 } $\begin{array}{c}\text { Triangle } \\
\text { Type }\end{array}$ & $4 \mathrm{msec}$ & $3 \mathrm{msec}$ & $2 \mathrm{msec}$ \\
\hline
\end{tabular}

(B) Results of Triangle Experiment (Uttal, 1971)

\begin{tabular}{llll} 
II & 95.7 & 86.7 & 78.7 \\
III & 80.8 & 65.9 & 48.2 \\
\hline
\end{tabular}

procedural features of the experiment were also the same. As previously, there was no fixation point. The $S$ was simply asked to say whether the randomly selected line was vertical or horizontal. A correct response level of $50 \%$, therefore, would represent chance performance. Each of the Ss was trained in three practice sessions prior to the collection of the tabulated data, The findings reported were collected in six experimental sessions-two sessions of $1 / 2 \mathrm{~h}$ duration for each of the three DVN levels.

The results of this experiment are presented in Table 1 as a function of thylocus of the stimulus lines and the noise density. Each value listed is based on between 1,300 and 1,500 random selections of that stimulus condition. As can be clearly seen from these tabulated results, there is only a very small measurable effect of retinal locus in this experiment, and even then it appears only for the denser 2-msec DVN levels. Specifically, while there was only a truly inconsequential $.6 \%$ difference between the $21 / 2-\mathrm{deg}$ and the $13 / 4-\mathrm{deg}$ loci for the $4-\mathrm{msec}$ condition and an equally insignificant $.4 \%$ difference for the 3-msec condition, the data tabulated in Table 1 do show that there is a $3.1 \%$ locus effect for the 2-msec DVN level. This latter value is but one-tenth of the difference encountered for the equivalent condition in our original experiment (Uttal, 1971).2 The second part of Table 1 shows the equivalent values for the Type II (corners missing) and the Type III (sides missing) triangles in that earlier experiment.

Clearly the locus effect is, at the worst, a minor perturbation on the geometrical effect to which we had previously attributed the phenomenon, even in the 2-msec DVN condition in which it is most pronounced. The geometrical or organizational effect is, of course, mainly associated with the factors of dot numerosity and spacing and their influence on line recognizability, dimensions which were considered in detail in our earlier paper on line recognition (Uttal, Bunnell, \& Corwin, 1970).

In sum, both on a priori grounds implicit in the control afforded by having no fixation point as well as on the basis of the present test, there appears to be no substance to the assertion that a possible uncontrolled retinal locus effect played any substantial role in contaminating the results of the triangle experiment. There is either a very small locus effect, or our experimental design controlled it adequately. In either case, the conclusions of the earlier paper (Uttal, 1971) still seem valid. 
Whatever minor perturbing effects are introduced by the difference in retinal locus of the corners and sides of a triangle remain much smaller than the geometrical or organizational role played by the individual dot in the overall pattern.

\section{REFERENCES}

ALPERN, M. Introduction to movements of the eyes. In $\mathrm{H}$. Davson (Ed.), The eye. (2nd ed.) Vol. 3. Muscular mechanisms. New York: Academic Press, 1969. Pp. 1-3.
HERSHENSON, M. Perception of letter arrays as a function of absolute retinal locus. Journal of Experimental Psychology, 1969, 80, 201-202.

MENZER, G. W., \& THURMOND, J. B. Form identification in peripheral vision. Perception \& Psychophysics, 1970, 8, 205-209.

UTTAL, W. R. Masking of alphabetic character recognition by dynamic visual noise (DVN). perception \& Psychophysics, 1969, 6, 121-128.

UTTAL, W. R. A masking approach to the problem of form perception. Perception \& Psychophysics, 1971, 9, 296-298.

UTTAL, W. R., BUNNELL, L. M., \& CORWIN, S. On the detectability of straight lines in visual noise: An extension of French's paradigm into the millisecond domain. Perception \& Psychophysics, $1970,8,385-388$.

\section{NOTES}

1. The details of the dynamic visual noise experiment are spelled out most completely in Uttal (1969).

2 . I should note that I have discovered a minor typographical error in the caption of Fig. 3 of the original paper on triangles (Uttal, 1971a). Points plotted with " $O$ " were for the Type II triangles and those plotted with "\#" were for the Type V triangles, rather than vice versa as indicated. These two are the overlapping curves and, therefore, this error makes no appreciable difference. 